\title{
Approximate transverse feedback linearization under digital control
}

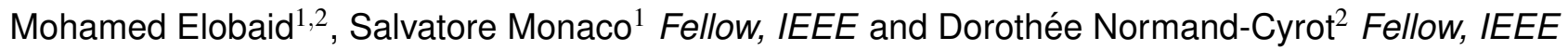

\begin{abstract}
Thanks to a suitable redesign of the maps involved in the continuous-time solution, a digital design procedure preserving transverse feedback linearization up to a prefixed order of approximation in the sampling period is described. Simulated examples illustrate the results.
\end{abstract}

Index Terms-Sampled-data control, Feedback linearization, Algebraic/geometric methods

\section{INTRODUCTION}

$\mathbf{S}$ EVERAL control problems, as for instance synchronization, path following or manoeuvring electro-mechanical systems, can be naturally cast in the context of set stabilization, relying on stabilization over surfaces that specify the control goals (e.g. [1],[2],[3],[4]). Various approaches have been proposed in the recent literature for solving set stabilization problems; among them the ones based on Lyapunov arguments (e.g. [1]), immersion and invariance (e.g. [5], [6]) and, more relevant for this manuscript, those based on the notion of feedback linearization (e.g. [7], [8]).

Generalizing the idea proposed in Banaszuk and Hauser [9] for solving a periodic orbit stabilization problem, Nielsen and Maggiore introduced in [2] the Transverse feedback linearization - TFL - approach. The underlying idea is to make the closed sub-manifold to be stabilized the zero-dynamics manifold associated to a suitable set of dummy output functions. Stabilization is then achieved by stabilizing the transverse linearized dynamics to this sub-manifold. The formalization and solution proposed in [2] provide an elegant framework for solving problems which can be reduced to constrain and control the evolutions over a suitable sub-manifold of the state space.

It is well known that in a digital context, i.e. under piecewise constant controls and periodical sampled measures, holding the continuous-time feedback solution constant over the sampling intervals significantly degrades the performances as the sampling period increases. On the other hand, a direct digital design based on the sampled-data model suffers from

Supported by Université Franco-Italienne/Università Italo-Francese (Vinci Grant 2019) .

${ }^{1}$ Dipartimento di Ingegneria Informatica, Automatica e Gestionale A. Ruberti (Sapienza University of Rome); Via Ariosto 25, 00185 Rome, Italy \{mohamed.elobaid, salvatore.monaco\} euniromal. it.

${ }^{2}$ Laboratoire de Signaux et Systèmes, L2S, CNRS, Univ. Paris Saclay; 3, Rue Joliot Curie, 91192, Gif-sur-Yvette, France \{mohamed.elobaid, dorothee.normand-cyrot \}ecentralesupelec.fr limitations induced by the loss of crucial control properties under sampling (e.g. feedback equivalence and zero-dynamics stability [10],[11]). Consequently, ad hoc schemes and design procedures are needed [12],[13].

In this work, under the assumption that a continuous-time feedback exists, we propose a digital solution that preserves transverse feedback linearization in an approximate sense. The result is achieved through a suitable sampling-dependent redesign of the functions which define the invariant submanifold. The procedure is based on the generalization to set stabilization of multi-input multi-output (MIMO) dynamics, of an iterative approach proposed in [14] for preserving the relative degree under sampling.

The paper is organized as follows. Section II recalls the continuous-time solution, motivates and sets the problem. The proposed control strategy is developed in Section III in a constructive way. Simulated examples are discussed in Section IV. Concluding remarks end the manuscript.

Notations: Functions and vector fields are assumed smooth and complete. Given $f$ and $g$, vector fields on $\mathbb{R}^{n}, \mathrm{~L}_{f}$ denotes the first order differential operator $\mathrm{L}_{f}=\sum_{i=1}^{n} f_{i}(\cdot) \frac{\partial}{\partial x_{i}}, \mathrm{~L}_{f} \mathrm{~L}_{g}$ their composition, $e^{\mathrm{L}_{f}}:=\mathrm{Id}+\sum_{i \geq 1} \frac{\mathrm{L}_{f}^{i}}{i !}$ the exponential Lie series operator with Id the identity operator and $\mathrm{L}_{f}^{i}$ iterative composition $i$ times of $\mathrm{L}_{f}$. Given a real valued function $h(\cdot)$ on $\mathbb{R}^{n},\left.e^{\mathrm{L}_{f}} h(x)\right|_{x(k)}$ denotes the application of the Lie series operator $e^{\mathrm{L}_{f}}$ to the function $h(x)$ evaluated at the state $x(k)$ so recovering the equality $\left.e^{\mathrm{L}_{f}} h(x)\right|_{x(k)}=h\left(e^{\mathrm{L}_{f}}(x(k))\right.$ where for simplicity one writes $\left.e^{\mathrm{L}_{f}}(x)\right|_{x(k)}=e^{\mathrm{L}_{f}}(x(k))$. $I_{r}$ indicates the identity square matrix of order $r$. Given a manifold $M$ and a closed connected set $N \subset M, N$ is said to be invariant under the dynamics $\dot{x}=f(x)+g(x) u$ if $\forall x_{0} \in N$ and any control $u(\cdot)$, $x(t) \in N, \forall t . N$ is controlled invariant if there exists a feedback $u^{\star}$ making $N$ invariant for the closed loop system. Given a pair of matrices $(A, B)$, one sets $\operatorname{col}(A, B)=\left(\begin{array}{ll}A^{\top} & B^{\top}\end{array}\right)^{\top}$ and similarly, blkdiag $\left(A_{i}\right)$ the block diagonal matrix formed by the matrices $A_{i}$. A continuous function $\beta(\cdot):[0, \infty) \rightarrow[0, \infty)$, that is zero at zero and strictly increasing and unbounded is said to be of class $\kappa_{\infty}$. A continuous function $R(x, \delta)$ is of order $O\left(\delta^{p}\right)$ with $p \geq 1$ if, whenever it is defined, it can be written as $R(x, \delta)=\delta^{p-1} \tilde{R}(x, \delta)$ and there exists a function $\beta(\delta) \in \kappa_{\infty}$ and $\delta^{\star}>0$ such that $\forall \delta \leq \delta^{\star},|\tilde{R}(x, \delta)| \leq \beta(\delta)$. Given $x_{0} \in M$, $U$ denotes a connected neighbourhood of $x_{0}$ in $M$. 


\section{Preliminaries and ReCAlls}

We consider the input-affine dynamics defined on $\mathbb{R}^{n}$

$$
\dot{x}=f(x)+\sum_{i=1}^{m} g_{i}(x) u_{i}=f(x)+g(x) u
$$

with $u \in \mathbb{R}^{m}$ and independent vector fields $g_{1}(x), \ldots, g_{m}(x)$.

\section{A. Recalls on transverse feedback linearization}

Transverse feedback linearization essentially refers to equivalence under feedback to a system characterized by a linear controllable sub-dynamics transverse to a given closed, controlled invariant, embedded sub-manifold in the system statespace. Formally from [2], one sets the problem below.

Problem 2.1: (L)TFL - Let $\Gamma^{\star} \subset \mathbb{R}^{n}$ be a closed, controlled invariant sub-manifold for dynamics (1) and let $x_{0} \in \Gamma^{\star}$; TFL is said to be locally solvable if there exist a feedback $u=\gamma(x, v)$ and a coordinates change $\phi(x)=\operatorname{col}\left(\phi_{1}(x), \phi_{2}(x)\right): x \mapsto(\xi, z)$, defined in a neighbourhood $U$ of $x_{0}$, such that (1) rewrites

$$
\begin{aligned}
& \dot{\xi}=A \xi+B v_{1} \\
& \dot{z}=f_{z}(\xi, z)+g_{z}^{1}(\xi, z) v_{1}+g_{z}^{2}(\xi, z) v_{2}
\end{aligned}
$$

where $\xi \in \mathbb{R}^{n-n^{\star}}, z \in \mathbb{R}^{n^{\star}}, v=\operatorname{col}\left(v_{1}, v_{2}\right) \in \mathbb{R}^{m}, n^{\star}=\operatorname{dim}\left(\Gamma^{\star}\right)$, $g_{z}^{1}(\cdot), g_{z}^{2}(\cdot)$ are smooth (matrix) valued functions, $B$ full column rank, the pair $(A, B)$ controllable, $\phi\left(\Gamma^{\star} \cap U\right)=\{(\xi, z)$ : $\xi=0\}$. When $U$ is a tubular neighbourhood of the whole $\Gamma^{\star}$, then TFL is said to hold globally.

The following comments are in order :

- $\dot{\xi}=A \xi+B v_{1}$ specifies the transverse dynamics and $v_{1}$ the transverse control;

- the dynamics of $z$, restricted to $\phi\left(\Gamma^{\star} \cap U\right), \dot{z}=f_{z}(0, z)+$ $g_{z}^{2}(0, z) v_{2}$, is referred to as the tangential dynamics with $v_{2}$ the tangential control;

- setting $v_{1}=-K \xi$ for a suitable $K$, (local) stabilization of $\Gamma^{\star}$ is achieved. If the trajectories of the closed loop system (under $v_{1}$ ) are bounded, stabilization of $\Gamma^{\star}$ holds.

Thanks to the decoupling of the control components, one independently forces the state evolutions towards $\Gamma^{\star}$ under $v_{1}$ and assigns a desired behaviour over it through $v_{2}$.

Before stating the necessary and sufficient conditions solving the (L)TFL problem given in [2], the well known notion of well defined vector relative degree is recalled [7].

Definition 2.1: The dynamics (1), with output $y=$ $\operatorname{col}\left(h_{1}(x), \ldots, h_{q}(x)\right), q \leq m, h_{i}(\cdot): \mathbb{R}^{n} \rightarrow \mathbb{R}$ has well-defined vector relative degree $r=\left(\begin{array}{lll}r_{1} & \ldots & r_{q}\end{array}\right)$ at $x_{0}$ if $L_{g_{j}} L_{f}^{k} h_{i}(x)=0$ on $U$ for $k=1, \ldots, r_{i}-2, i=1, \ldots, q, j=1, \ldots, m$, while $L_{g_{j}} L_{f}^{r_{i}-1} h_{i}\left(x_{0}\right) \neq 0$ for some $j$ and the $(q \times m)$ decoupling matrix $[D(x)]_{i, j}=L_{g_{j}} L_{f}^{r_{i}-1} h_{i}(x)$ is full rank at $x_{0} ; r$ is globally defined if the rank condition holds on $\mathbb{R}^{n}$ with respect to a fixed decoupling sub-matrix.

Theorem 2.1: ([2], [15]) The LTFL Problem 2.1 is solvable if and only if there exist $\rho$ smooth $\mathbb{R}$-valued functions $\left(\alpha_{1}(x), \ldots, \alpha_{\rho}(x)\right), \rho \leq m$, defined on $U$ such that:

1) $\Gamma^{\star} \cap U \subset\left\{x \in U: \alpha_{i}(x)=0, i=1, \ldots \rho\right\}$;

2) the dynamics (1) with output $\alpha(x)=$ $\operatorname{col}\left(\alpha_{1}(x), \ldots, \alpha_{\rho}(x)\right)$, has a well defined vector relative degree $r=\left(r_{1}, \ldots, r_{\rho}\right)$ at $x_{0}$ with $\sum_{i=1}^{\rho} r_{i}=n-n^{\star}$.
When $\phi(x)$ and $\alpha(x)$ are defined everywhere in a tubular neighbourhood of $\Gamma^{\star}$, then TFL holds globally.

Theorem 2.1 specifies that Problem 2.1 is indeed equivalent to partial feedback linearization and zero dynamics assignment with respect to a suitable dummy output vector.

Remark 2.1: Finding the functions $\alpha_{i}, i=1, \ldots, \rho$, from the given control specifications may be a difficult task. A procedure based on the annihilator of some controlled invariant distributions coinciding with the tangent bundle to $\Gamma^{\star}$ is developed in [2].

Without loss of generality, we assume in the sequel that the matrices $(A, B)$ are in the Brunowsky canonical form with

$$
\begin{aligned}
A & :=\operatorname{blkdiag}\left(A_{1}, \ldots, A_{\rho}\right) \in \mathbb{R}^{n-n^{\star}} \times \mathbb{R}^{n-n^{\star}} \\
B & :=\operatorname{blkdiag}\left(B_{1}, \ldots, B_{\rho}\right) \in \mathbb{R}^{\left(n-n^{\star}\right) \times m} \\
A_{i} \in \mathbb{R}^{r_{i} \times r_{i}} & =\left(\begin{array}{ccccc}
0 & 1 & 0 & \ldots & 0 \\
0 & 0 & 1 & \ldots & 0 \\
& & \vdots & & \\
0 & 0 & 0 & \ldots & 1 \\
0 & 0 & 0 & \ldots & 0
\end{array}\right), B_{i} \in \mathbb{R}^{r_{i} \times 1}=\left(\begin{array}{c}
0 \\
0 \\
\vdots \\
0 \\
1
\end{array}\right) .
\end{aligned}
$$

\section{B. Recalls on sampled-data dynamics}

The sampled-data model we are dealing with is characterized by a dynamics for which the measures are available at periodic sampling instants and the controls kept constant over the sampling period. This naturally arises in presence of digital actuation and sensing devices. More precisely in the present paper, dynamics (1) is fed by constant controls over time intervals of length $\delta$, i.e. $u_{i}(t)=u_{i}(k \delta)=u_{i}(k)$ for $t \in[k \delta,(k+1) \delta[, k \geq 0$. Accordingly, the sampled-data dynamics equivalent to (1) takes the form

$$
\begin{aligned}
x(k+1) & =F^{\delta}(x(k), u(k))=e^{\delta\left(\mathrm{L}_{f}+\sum_{i=1}^{m} u_{i}(k) \mathrm{L}_{g_{i}}\right)}(x(k)) \\
& =x(k)+\sum_{j \geq 1} \frac{\delta^{j}}{j !}\left(\mathrm{L}_{f}+\sum_{i=1}^{m} u_{i}(k) \mathrm{L}_{g_{i}}\right)^{j}(x(k))
\end{aligned}
$$

with $u(k)=\operatorname{col}\left(u_{1}(k), \ldots, u_{m}(k)\right)$ and the function $F^{\delta}(\cdot, u)$ defined by its series expansion in powers of $\delta$, [16]. When truncating each row of this expansion at any fixed order $\operatorname{col}\left(p_{1}, \ldots, p_{n}\right)$ in $\delta$, so neglecting row-wise the remaining terms in $\left(O\left(\delta^{p_{1}+1}\right), \ldots, O\left(\delta^{p_{n}+1}\right)\right)$ in the infinite series expansion, the sampled-data model is said to be approximated at the order $\operatorname{col}\left(p_{1}, \ldots, p_{n}\right)$. Given a real valued output function $y_{i}=h_{i}(x)$, starting from $x(k)$ at time $t=k \delta$, one computes the output at any sampling instant $t=(k+j) \delta, j>0$, under the control sequence $(u(k), \ldots, u(k+j-1))$, through the usual composition of functions so getting

$$
\begin{aligned}
y_{i}(k+j) & =h_{i}(x(k+j)) \\
& =h_{i} \circ F^{\delta}(\cdot, u(k+j-1)) \circ \ldots \circ F^{\delta}(x(k), u(k)) .
\end{aligned}
$$

For sampled-data dynamics, the notion of relative degree reads as follows.

Definition 2.2: [16] The MIMO sampled data dynamics (4) with output vector $y=\operatorname{col}\left(h_{1}(x), \ldots, h_{q}(x)\right)$ has a well defined 
vector relative degree $r=\left(r_{1}, \ldots, r_{q}\right)$ at $x_{0}$, if the following holds true for $x \in U, i=1, \ldots, q$

$$
\begin{array}{ll}
\frac{\partial y_{i}(l)}{\partial u_{j}(0)}(x)=0, & l=1, \ldots r_{i}-1 ; j=1, \ldots m \\
\frac{\partial y_{i}\left(r_{i}\right)}{\partial u_{j}(0)}\left(x_{0}\right) \neq 0 \quad & \text { for some } j
\end{array}
$$

and the sampled data $(q \times m)$ decoupling matrix $\left[D^{\delta}(x)\right]_{i, j}=$ $\frac{\partial y_{i}\left(r_{i}\right)}{\partial u_{j}(0)}(x)$ is full rank at $x_{0}$. If the $p_{i}$ 's are the highest orders of the expansions in power of $\delta$ at which the conditions above hold, the system (4) is said to have a vector relative degree $r=\left(r_{1}, \ldots, r_{q}\right)$ at $x_{0}$ at the order $p=\operatorname{col}\left(p_{1}, \ldots, p_{q}\right)$ in $\delta$ (truncation with error in $\operatorname{col}\left(O\left(\delta^{p_{1}+1}\right), \ldots, O\left(\delta^{p_{q}+1}\right)\right)$.

The falling to one of the relative degree is a well known fact, which is emblematic of the appearance of extra sampling zero-dynamics responsible for serious limitations in feedback design (see [17] for the linear framework and (e.g. [11],[18]) for the nonlinear one). The following result from [16] is recalled.

Lemma 2.1: Given dynamics (1) with output vector $y=$ $\operatorname{col}\left(h_{1}(x), \ldots, h_{q}(x)\right)$ and well defined vector relative degree at $x_{0}$ then, there exists $T^{\star}>0$ such that for any $\left.\delta \in\right] 0, T^{*}[$, its sampled-data equivalent model (4) has well defined vector relative degree equal to $r=(1, \cdots, 1)$ at $x_{0}$, whenever one takes into account for each respective output $y_{i}$, approximations in $\delta$ of order at least $r_{i}$, the continuous-time relative degree. The result easily follows from (4) by computing

$$
\frac{\partial y_{i}(1)}{\partial u_{j}(0)}=\left.\frac{\delta^{r_{i}}}{r_{i} !} L_{g_{j}} L_{f}^{r_{i}-1} h_{i}(x)\right|_{x_{0}}+O\left(\delta^{r_{i}+1}\right)
$$

because $\forall i=(1, \ldots, q), L_{g_{j}} L_{f}^{r_{i}-1} h_{i}\left(x_{0}\right) \neq 0$ for some $j$.

\section{Problem statement}

Assuming that a solution to the (L)TFL Problem 2.1 exists, does a sampled-data solution exist ? How to compute it and what about its performances ? With this in mind and recalling that, because of (3), the coordinates $\xi$ in Theorem 2.1 are

$$
\xi=\operatorname{col}\left(\xi_{1}, \ldots, \xi_{\rho}\right) \quad \text { with } \quad \xi_{i}=\operatorname{col}\left(\alpha_{i}, \ldots, L_{f}^{r_{i}-1} \alpha_{i}\right)(x)
$$

the sampled-data (L)TFL problem is set.

Problem 2.2: (SD-(L)TFL) Given dynamics (1) satisfying the conditions of Theorem 2.1, find for any $\delta \in] 0, T^{\star}\left[, T^{*}>0\right.$ small enough, a piecewise constant feedback $u^{\delta}=\gamma^{\delta}(x, v)$ and a coordinates change $\phi^{\delta}(\cdot): x \mapsto\left(\xi^{\delta}, z\right)$ defined in a neighbourhood $U$ of $x_{0} \in \Gamma^{\star}$, under which the sampled-data closed loop dynamics takes the normal form below

$$
\begin{aligned}
\xi^{\delta}(k+1) & =(I d+\delta A) \xi^{\delta}(k)+\delta B v_{1}(k) \\
z(k+1) & =F_{z}^{\delta}\left(\xi^{\delta}(k), z(k), v(k)\right)
\end{aligned}
$$

with $\xi^{\delta} \in \mathbb{R}^{n-n^{\star}}, \xi^{\delta}=\operatorname{col}\left(\xi_{1}^{\delta}, \ldots, \xi_{\rho}^{\delta}\right), z \in \mathbb{R}^{n^{\star}}, v=$ $\operatorname{col}\left(v_{1}, v_{2}\right) \in \mathbb{R}^{m}$ and $\phi^{\delta}\left(\Gamma^{\star} \cap U\right)=\left\{\left(\xi^{\delta}, z\right): \xi^{\delta}=0\right\}=$ $\{(\xi, z): \xi=0\}=\phi\left(\Gamma^{\star} \cap U\right)$. The approximate SD-(L)TFL is solvable at degree $p \geq 1$, if (7a) is approximated in $O\left(\Delta^{p+1}\right)$

$$
\xi^{\delta}(k+1)=(I d+\delta A) \xi^{\delta}(k)+\delta B v_{1}(k)+O\left(\Delta^{p+1}\right)
$$

with $\Delta^{p+1}=\operatorname{col}\left(\boldsymbol{\delta}^{r_{1}+p}, \ldots, \boldsymbol{\delta}^{p+1}, \ldots, \boldsymbol{\delta}^{r_{\rho}+p}, \ldots, \boldsymbol{\delta}^{p+1}\right)$. If the relative degree is well defined for all $x \in \Gamma^{*}$ we will say, with a little abuse of nomenclature, that the problem is globally solved around $\Gamma^{*}$.

Some remarks are in order.

- Problem 2.2 should be understood as the preservation of the (L)TFL property under sampling. It does not concern the existence and the computation, for the equivalent sampled-data dynamics, of a closed controlled invariant sub-manifold. This data of the design is assumed to be known from the continuous-time solution. Note that, with a little abuse, the same notation, $U$, is used to denote the set over which the continuous-time and the sampled-data solutions are defined.

- The sampled-data tangential $z$-dynamics on $\Gamma^{\star}$ (setting $\xi^{\delta}=0$ ) is not constrained and recovers the continuoustime $z$-dynamics in $O\left(\delta^{2}\right)$.

- The (L)TFL solution described in Theorem 2.1 relies on partial feedback linearization with respect to outputs with suitable well defined vector relative degrees. It is clear from Lemma 2.1 that (L)TFL is lost under sampling except when these relative degrees are all equal to 1 .

- The approximation in (8) must be understood as an approximation at the $\Delta^{p}$-vector's order; i.e. the $j^{\text {th }}$ component of $\xi_{i}^{\delta}=\operatorname{col}\left(\xi_{i, 1}^{\delta}, \ldots, \xi_{i, r_{i}}^{\delta}\right)$ in $\xi^{\delta}$, is approximated at the order $\left(p+r_{i}-j\right)$. Such a non homogeneous approximation reflects the preservation of each relative degree $r_{i}$ of the respective $\xi_{i}^{\delta}$ at an order of approximation that has to be at least $r_{i}$ itself. This is at the basis of the result here proposed and reveals to be profitable in the achieved performances illustrated through simulations.

\section{ApPRoximate tRANSVERSE FEEDBACK LINEARIZATION UNDER DIGITAL CONTROL}

In this section, making use of a suitable sampling-dependent redesign of the output functions, we propose an approximated solution which combines computational simplicity with a significant improvement with respect to the zero-order-holding implementation of the continuous-time control law.

Let us start by pointing out why the continuous-time design approach cannot be directly applied in the sampleddata context. As noted before, the continuous-time solution is achieved by stabilizing $\Gamma^{*}$, rendered the zero dynamics submanifold of (1) with respect to a set of $(\rho \leq m)$ suitably chosen dummy output functions, the $\alpha_{i}(\cdot)$ 's, that have a well defined vector relative degree $r=\left(r_{1}, \ldots, r_{\rho}\right)$. The control goal is then assured under input-to-output feedback linearization and linear stabilization. Such an approach cannot be applied to the sampled-data model as $\Gamma^{*}$, the subset of the state-space to stabilize and to make invariant, is not a zero dynamics sub-manifold of the sampled-data model (4) associated to the $\alpha_{i}(\cdot)$ 's. Moreover, because of Lemma 2.1, even the dimension of the sampled-data zero dynamics sub-manifold is not the same as that of $\Gamma^{*}$. The main result stated below, relying on the extension to MIMO systems of a procedure proposed in [14], shows how starting from the $\alpha_{i}(\cdot)$ 's, a new set of delta-dependent functions, the $\alpha_{i}^{\delta}(\cdot)$ 's , can be designed for preserving the relative degrees and, at the same time, the zero dynamics sub-manifold. In addition, by working on the 
approximated sampled-data model it is shown that solutions, at increasing degrees of approximation can be computed.

With this in mind, under the conditions of Theorem 2.1, in the coordinates $\xi=\phi_{1}(x)$ as in (6) and $z=\phi_{2}(x)$, with $\operatorname{col}\left(\phi_{1}(x), \phi_{2}(x)\right)$ defining a smooth deffeomeorphism, (1) takes the form

$$
\begin{aligned}
\dot{\xi} & =A \xi+B\left(f_{a}(\xi, z)+g_{a}(\xi, z) u\right) \\
\dot{z} & =f_{z}(\xi, z)+g_{z}(\xi, z) u
\end{aligned}
$$

with $\xi=\operatorname{col}\left(\xi_{1}, \ldots, \xi_{\rho}\right) \in \mathbb{R}^{n-n^{\star}}, z \in \mathbb{R}^{n^{\star}}, \quad \xi_{i}=$ $\operatorname{col}\left(\xi_{i, 1}, \ldots, \xi_{i, r_{i}}\right) \in \mathbb{R}^{r_{i}}, \quad(A, B)$ as in (3), $B\left(f_{a}(\xi, z)+\right.$ $\left.g_{a}(\xi, z) u\right):=\operatorname{col}\left(B_{1}\left(f_{a_{1}}+g_{a_{1}} u\right), \ldots, B_{\rho}\left(f_{a_{\rho}}+g_{a_{\rho}} u\right)\right) \in$ $\mathbb{R}^{\left(n-n^{\star}\right) \times m}, f_{a}=\operatorname{col}\left(f_{a_{1}}, \ldots, f_{a_{\rho}}\right), g_{a}(\xi, z)=\operatorname{col}\left(g_{a_{1}}, \ldots, g_{a_{\rho}}\right)$, $g_{a_{i}}=\left(g_{a_{i}}^{1}, \ldots, g_{a_{i}}^{m}\right)$ and

$$
\begin{aligned}
& f_{a_{i}}(\xi, z)=\left.L_{f(x)}^{r_{i}} \xi_{i}^{1}\right|_{x=\phi^{-1}(\xi, z)}, \quad i=1, \ldots, \rho \\
& g_{a_{i}}^{j}(\xi, z)=\left.L_{g_{j}(x)} L_{f(x)}^{r_{i}-1} \xi_{i}^{1}\right|_{x=\phi^{-1}(\xi, z)}, i=1, \ldots, \rho, j=1, \ldots, m \\
& f_{z}(\xi, z)+g_{z}(\xi, z) u=\left.\frac{\partial \phi_{2}}{\partial x}(f(x)+g(x) u)\right|_{x=\phi^{-1}(\xi, z)} .
\end{aligned}
$$

In these coordinates, the sampled-data dynamics equivalent to (9) reads as (dropping the $k$-index in the right hand side)

$$
\begin{aligned}
\xi(k+1) & =A^{\delta} \xi+B_{1}^{\delta}\left(f_{a}(\xi, z)+g_{a}(\xi, z) u\right)+O\left(\Delta^{2}\right) \\
z(k+1) & =z+\delta\left(f_{z}(\xi, z)+g_{z}(\xi, z) u\right)+O\left(\delta^{2}\right)
\end{aligned}
$$

with $A^{\delta}=\operatorname{blkdiag}\left(A_{1}^{\delta}, \ldots, A_{\rho}^{\delta}\right), B_{j}^{\delta}=\operatorname{blkdiag}\left(B_{j, 1}^{\delta}, \ldots, B_{j, \rho}^{\delta}\right)$ with $A_{i}^{\delta}=e^{\delta A_{i}}$ and $B_{1, i}^{\delta}=\int_{0}^{\delta} e^{\tau A_{i}} B_{i} d \tau, B_{j, i}^{\delta}=\sum_{k \geq j} \frac{\delta^{k} !}{k !}\left(A_{i}\right)^{k-j} B_{i}$, so getting

$$
A_{i}^{\delta}=\left(\begin{array}{ccccc}
1 & \delta & \frac{\delta^{2}}{2 !} & \ldots & \frac{\delta^{r_{i}-1}}{\left(r_{i}-1\right) !} \\
0 & 1 & \delta & \ldots & \frac{\delta_{i}-2}{\left(r_{i}-2\right) !} \\
& & \vdots & & \\
0 & 0 & 0 & \ldots & 1
\end{array}\right), \quad B_{j, i}^{\delta}=\left(\begin{array}{c}
\frac{\delta^{r_{i}+j-1}}{\left(r_{i}+j-1\right) !} \\
\vdots \\
\frac{\delta^{j}}{j !}
\end{array}\right) .
$$

The main result can now be stated.

Theorem 3.1: Under the conditions of Theorem 2.1, there exist a small enough $T^{\star}>0$, and for any $\left.\delta \in\right] 0, T^{\star}[$, a $\delta$-dependent coordinates change $\phi^{\delta}(x)$ and a sampled-data feedback $\gamma^{\delta}(x, v)$ solving Problem 2.2 in $O\left(\Delta^{2}\right)$.

Proof: Under the assumption of well-defined vector relative degree and without loss of generality (possibly after a reordering of the control variables in $\xi), g_{a}(\xi, z)$ in (9) takes the form of a full rank matrix $D(\xi, z)=[M(\xi, z) \quad N(\xi, z)]$ with $M(\xi, z), \rho \times \rho$ full rank, and $N(\xi, z)$ spanning $\operatorname{ker}(D(\xi, z))$. Thus, with a little abuse of notations, the continuous-time normal form rewrites as

$$
\begin{aligned}
\dot{\xi} & =A \xi+B\left(f_{a}(\xi, z)+M(\xi, z) u_{1}+N(\xi, z) u_{2}\right) \\
\dot{z} & =f_{b}(\xi, z)+g_{b}(\xi, z) u
\end{aligned}
$$

with $u=\operatorname{col}\left(u_{1}, u_{2}\right), u_{1} \in \mathbb{R}^{\rho}, u_{2} \in \mathbb{R}^{m-\rho}$. Accordingly, the linearizing continuous-time feedback reads as

$$
\begin{aligned}
u_{1} & =\gamma_{1}\left(\xi(k), z(k), v_{1}(k), u_{2}(k)\right) \\
& =M^{-1}(\xi, z)\left(-f_{a}(\xi, z)-N(\xi, z) u_{2}(k)+v_{1}(k)\right)
\end{aligned}
$$

with external control vector $v_{1} \in \mathbb{R}^{\rho}$. The second part of the proof stands in the computation of a reshaped $\delta$ dependent dummy output, $\alpha^{\delta}(x)=\operatorname{col}\left(\alpha_{1}^{\delta}(x), \ldots, \alpha_{\rho}^{\delta}(x)\right)$, under which the vector relative degree $r=\left(r_{1}, \ldots, r_{\rho}\right)$ is preserved under sampling, up to approximations in $\operatorname{col}\left(O\left(\delta^{r_{1}+1}\right), \ldots, O\left(\delta^{r_{\rho}+1}\right)\right)$. Moreover, the zero dynamics sub-manifold is preserved at the same orders of approximation. For, let us associate to each function $\alpha_{i}, i=1, \ldots, \rho$

$$
\alpha_{i}^{\delta}(x)=\alpha_{i}(x)+\sum_{j=1}^{r_{i}-1} \delta^{j} c_{i, j} \alpha_{i}^{(j)}(x)=C_{i}^{\delta} \xi_{i}(x)
$$

where $\alpha_{i}^{(j)}=L_{f}^{j}\left(\alpha_{i}\right)$ is the $j^{\text {th }}$-time derivative of $\alpha_{i}$ and the real coefficients $c_{i, j}$ are the entries of the row matrix

$$
\begin{aligned}
C_{i}^{\delta} & =\operatorname{col}\left(1, \delta c_{i, 1}, \ldots, \delta^{r_{i}-1} c_{i, r_{i}-1}\right) \\
& =\delta^{r_{i}} B_{i}^{T}\left(\begin{array}{llll}
B_{1, i}^{\delta} & A_{i}^{\delta} B_{1, i}^{\delta} & \ldots & \left.\left(A_{i}^{\delta}\right)^{\left(r_{i}-1\right)} B_{1, i}^{\delta}\right)^{-1}
\end{array}\right.
\end{aligned}
$$

with $A_{i}^{\delta}$ and $B_{1, i}^{\delta}$ as in (11). The so defined $\delta$-dependent functions are used to define the first $\left(r_{1}+\cdots+r_{\rho}\right)$ coordinates of a block diagonal transformation to be applied to the approximated sampled-data representation (10). For, we define

$$
\phi^{\delta}(x)=\operatorname{col}\left(\xi^{\delta}, z\right):=\left(\begin{array}{cc}
T_{n-n^{\star}}(\delta) & 0 \\
0 & I_{n^{\star}}
\end{array}\right)\left(\begin{array}{l}
\xi \\
z
\end{array}\right)
$$

with $T_{n-n^{\star}}(\boldsymbol{\delta})=\operatorname{blkdiag}\left(T_{1}(\boldsymbol{\delta}), \ldots, T_{\rho}(\boldsymbol{\delta})\right) \in \mathbb{R}^{\left(n-n^{\star}\right) \times\left(n-n^{\star}\right)}$

$$
\xi_{i}^{\delta}(x)=T_{i}(\delta) \xi_{i}(x):=\left(\begin{array}{c}
C_{i}^{\delta} \\
\frac{1}{\delta} C_{i}^{\delta}\left(A_{i}^{\delta}-I_{r_{i}}\right) \\
\vdots \\
\frac{1}{\delta^{r_{i}-1}} C_{i}^{\delta}\left(A_{i}^{\delta}-I_{r_{i}}\right)^{\left(r_{i}-1\right)}
\end{array}\right) \xi_{i}(x) .
$$

It is a matter of computation to verify that the vector relative degree of the functions (13) is $r=\left(r_{1}, \ldots, r_{\rho}\right)$ and that the feedback (12), with $v_{1}^{\delta}$ in the place of $v_{1}$, transforms (10) into

$$
\begin{array}{r}
\xi^{\delta}(k+1)=\left(I_{\left(n-n^{*}\right)}+\delta A\right) \xi^{\delta}(k)+\delta B v_{1}^{\delta}(k)+O\left(\Delta^{2}\right) \\
z(k+1)=z(k)+\delta\left(f_{b}(\xi, z)+g_{b}(\xi, z) \operatorname{col}\left(v_{1}^{\delta}, u_{2}\right)+O\left(\delta^{2}\right) .\right.
\end{array}
$$

Hence, the posed problem is solved at the fixed approximated order. Moreover, setting $v_{1}^{\delta}(k)=-K \xi^{\delta}(k)$, with gains $k_{i, j}$ 's, $i=1, \ldots, \rho, j=1, \ldots, n-n^{*}$, suitably chosen to assign Hurwitz polynomials, stabilization of $\Gamma^{*}$ is attained at the same order of approximation, since the sampled-data zero dynamics manifold recovers the continuous-time one, i.e. $\phi^{\delta}\left(\Gamma^{\star} \cap U\right)=$ $\left\{\left(\xi^{\delta}, z\right) ; \xi^{\delta}=0\right\}=\{(\xi, z) ; \xi=0\}=\phi\left(\Gamma^{\star} \cap U\right)$. The sampleddata feedback so far designed is thus

$$
u_{1}^{\delta}(k)=\gamma_{1}^{\delta}\left(\xi(k), z(k),-K \xi^{\delta}(k), u_{2}(k)\right) .
$$

It provides a local solution around any point at which the vector relative degree is well defined; it provides a global solution around $\Gamma^{*}$ if the vector relative degree is defined at any point of $\Gamma^{*}$.

The approach could be further developed to increase the approximation order along the lines of the proof of Theorem 3.1 and extending the SISO procedure in [14]. Starting from an higher order approximation of the sampled-data model (10), the idea is to add a $\delta$-dependent part to the control law to compensate the effects of the additional terms occuring at the corresponding degree of approximation. Iteratively, a controller of the form $u_{1}(k)=u_{1,0}(k)+\delta u_{1,1}(k)+\cdots+\frac{\delta^{p}}{p !} u_{1, p}(k)$ is built, with $u_{1,0}$ as in (17), to reach a solution approximated 


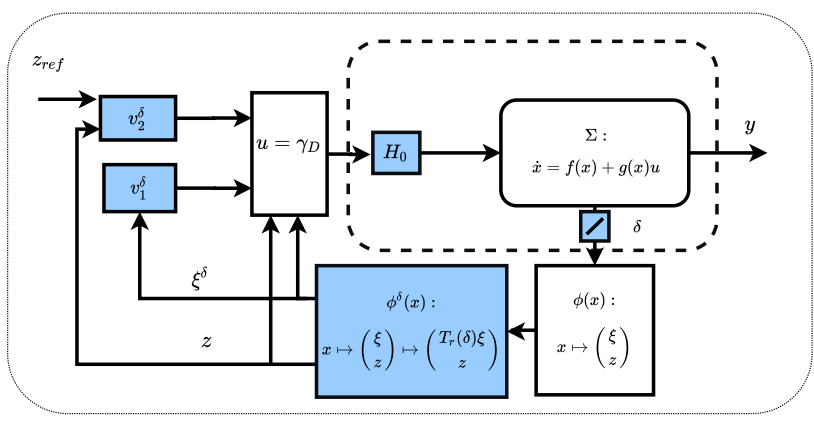

(a)

(a) Proposed SDTFL

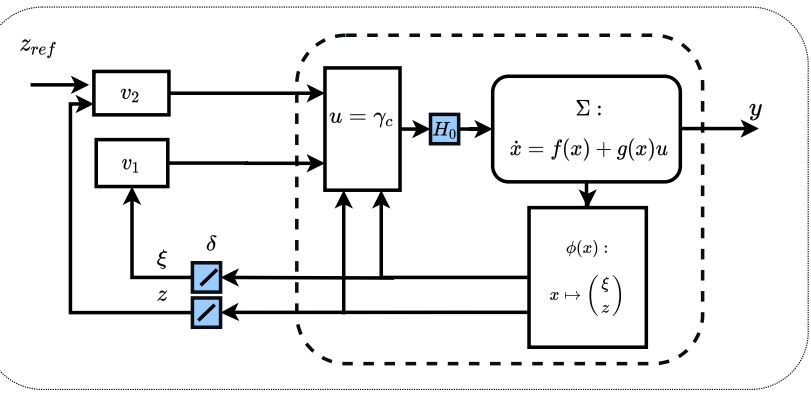

(b)

Fig. 1: Implementation of the proposed sampled-data solution (a), compared to zero-order-holding of the continuous-time solution (b). The modified coordinates change and feedback of the proposed solution are highlighted in blue.

in $O\left(\Delta^{p+1}\right)$. Detailed computations are left out for the sake of space.

Remark 3.1: It is worth to note that the controller (17) can be easily computed since $\xi^{\delta}=T(\delta) \xi$ depends on $r_{1}, \ldots, r_{\rho}$ only and can be precomputed offline. Each function $\alpha_{i}^{\delta}(\cdot)$ is a polynomial in the first $r_{i}$ time derivatives of $\alpha_{i}(\cdot)$, with real $\delta$-depend coefficients. For the first values of $r_{i}$, one computes

$$
\begin{gathered}
r_{i}=2: \quad \alpha_{i}^{\delta}=\alpha_{i}-\frac{\delta}{2} \dot{\alpha}_{i}=\xi_{i, 1}-\frac{\delta}{2} \xi_{i, 2} \\
r_{i}=3: \quad \alpha_{i}^{\delta}=\alpha_{i}+\delta \dot{\alpha}_{i}-\frac{\delta^{2}}{3} \ddot{\alpha}_{i}=\xi_{i, 1}+\delta \xi_{i, 2}-\frac{\delta^{2}}{3} \xi_{i, 3} \\
T_{2}(\delta)=\left(\begin{array}{cc}
1 & \frac{-\delta}{2} \\
0 & 1
\end{array}\right) ; \quad T_{3}(\delta)=\left(\begin{array}{ccc}
1 & \delta & -\frac{\delta^{2}}{3} \\
0 & 1 & \frac{-\delta}{2} \\
0 & 0 & 1
\end{array}\right) .
\end{gathered}
$$

\section{EXAMPLES}

In this section two examples from [2] and [19] are worked out to illustrate the benefits of the proposed digital design procedure. Figure 1 depicts the implementation schemes.

Example 4.1 (SISO): Consider the unicycle model

$$
\dot{x}=v \cos \theta, \quad \dot{y}=v \sin \theta, \quad \dot{\theta}=w
$$

with $v=1$, under the control goal of tracking a circular path $\left\{(x, y, \theta): x^{2}+y^{2}-1=0\right\}$. Following [19], one sets the transversal output as $\alpha(x, y, \theta)=x^{2}+y^{2}-1$, with relative degree $r=2=n-n^{\star}$ over $\mathbb{R}^{3} \backslash\left\{\theta=\tan ^{-1} \frac{y}{x}\right\}$. Then, under the coordinates change $\phi(x, y, \theta)=\operatorname{col}\left(x^{2}+y^{2}-1,2(x \cos \theta+\right.$ $y \sin \theta), \theta)=\left(\xi_{1}, \xi_{2}, z\right)$, the dynamics (18) is transformed into the normal form

$$
\dot{\xi}_{1}=\xi_{2} ; \quad \dot{\xi}_{2}=2(1+b(\xi, z) u) ; \quad \dot{z}=u
$$

with $b(\xi, z)=\left.2(y \cos \theta-x \sin \theta)\right|_{(x, y, \theta)=\phi(\xi, z)^{-1}}$. Under sampling, because $\left(n=3, n^{\star}=1, m=1\right)$, one reshapes the transversal output as in (13), so getting

$\alpha^{\delta}(x, y, \theta)=\alpha(x, y, \theta)-\frac{\delta}{2} \dot{\alpha}(x, y, \theta)=x^{2}+y^{2}-\delta(x \cos \theta+y \sin \theta)$.

Accordingly, under the coordinates change

$$
\phi^{\delta}(\cdot)=\operatorname{col}\left(\xi_{1}^{\delta}, \xi_{2}^{\delta}, z\right)=\operatorname{col}\left(T_{2}(\delta) \xi, z\right)=\operatorname{col}\left(\xi_{1}-\frac{\delta}{2} \xi_{2}, \xi_{2}, z\right)
$$

the sampled-data equivalent model to (19) is transformed into

$$
\begin{aligned}
\xi_{1}^{\delta}(k+1) & =\xi_{1}^{\delta}(k)+\delta \xi_{2}^{\delta}(k)+O\left(\delta^{3}\right) \\
\xi_{2}^{\delta}(k+1) & =\xi_{2}^{\delta}(k)+2 \delta(1+b(\xi, z) u(k))+O\left(\delta^{2}\right) \\
z(k+1) & =z(k)+\delta u(k) .
\end{aligned}
$$

Finally, the sampled-data feedback law

$$
u(k)=(2 b(\xi(k), z(k)))^{-1}\left(-2+v^{\delta}(k)\right)
$$

with $v^{\delta}(k)=-k_{1} \xi_{1}^{\delta}-k_{2} \xi_{2}^{\delta}$ and suitably chosen $k_{1}=$ $16.6, k_{2}=-11.1$, stabilizes the dynamics onto $\Gamma^{\star}\left(\xi^{\delta}=0\right)$. Setting $x_{0}=\left(-1.5,1, \frac{\pi}{4}\right)$, comparative simulations between the continuous-time CT design (in red), $\mathrm{ZOH}$ of the CT feedback (in black) and the sampled-data proposed solution (in blue) are plotted, for a sampling period $\delta=0.3$. As clearly illustrated through the simulations (Figure 2), the proposed design outperforms $\mathrm{ZOH}$ of the $\mathrm{CT}$ solution.

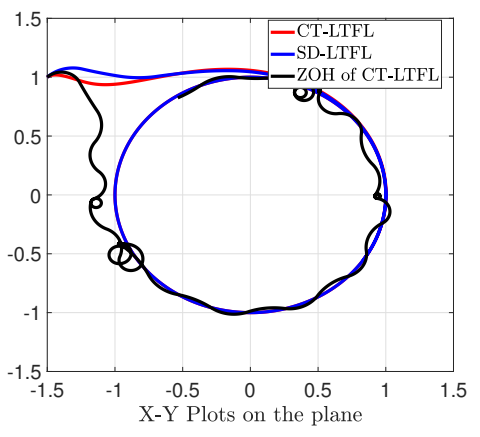

Fig. 2: SD feedback VS ZOH of the CT feedback $\delta=0.3$

Example 4.2: (MIMO) Let the input-affine dynamics

$$
\dot{x}=f(x)+g_{1}(x) u_{1}+g_{2}(x) u_{2}
$$

with $f(x)=\left(\begin{array}{llll}-x_{2} & x_{1} & x_{3} x_{4} & 0\end{array}\right)^{\top}, g_{1}(x)=\left(\begin{array}{llll}0 & 0 & x_{3} & 1\end{array}\right)^{\top}$, $g_{2}(x)=\left(\begin{array}{llll}-x_{2} & x_{1} & 0 & 0\end{array}\right)^{\top}$. The LTFLP has been set and solved in [2] with the control goal of reaching and traversing an elliptic paraboloid immersed in the subspace $x_{4}=0$; i.e. $\left\{x \in \mathbb{R}^{4}: x_{1}^{2}+x_{2}^{2}-x_{3}=x_{4}=0\right\}$ around $x_{0}=\operatorname{col}(4,0,2,0)$. Because $\left(n=4, n^{\star}=2, m=2\right)$, it has been shown that the function $\alpha(x)=\ln \left(\frac{x_{3}}{x_{1}^{2}+x_{2}^{2}}\right)-x_{4}$, has relative degree $2=n-n^{\star}$ over $\mathbb{R}^{4} /\left\{x \in \mathbb{R}^{4}: x_{1}=x_{2}=0 ; x_{3}=0\right\}$, so defining the coordinates 
change $\operatorname{col}\left(\xi_{1}, \xi_{2}, z\right)=\phi(x)=\operatorname{col}\left(\ln \left(\frac{x_{3}}{x_{1}^{2}+x_{2}^{2}}\right)-x_{4}, x_{4}, x_{1}, x_{2}\right)$ and $u^{\star}=(0,0)^{\top}$. Under sampling, setting according to (13) $\alpha^{\delta}(x)=\alpha(x)-\frac{\delta}{2} \dot{\alpha}(x)$, one defines the sampled coordinates change as $\operatorname{col}\left(\xi_{1}^{\delta}, \xi_{2}^{\delta}, z\right)=\phi^{\delta}(x)=\operatorname{col}\left(\phi_{1}^{\delta}(x), x_{1}, x_{2}\right)$, with

$$
\phi_{1}^{\delta}(x)=\operatorname{col}\left(\ln \left(\frac{x_{3}}{x_{1}^{2}+x_{2}^{2}}\right)-\left(1+\frac{\delta}{2}\right) x_{4}, x_{4}\right)=T_{2}(\delta) \operatorname{col}(\alpha(x), \dot{\alpha}(x))
$$

under which (21) is transformed, after sampling, into

$$
\begin{aligned}
\xi_{1}^{\delta}(k+1) & =\xi_{1}^{\delta}(k)+\delta \xi_{2}^{\delta}(k) \\
\xi_{2}^{\delta}(k+1) & =\xi_{2}^{\delta}(k)+\delta u_{1}(k) \\
z_{1}(k+1) & =z_{1}(k)-\delta z_{2}(k)\left(1+u_{2}(k)\right)+O\left(\delta^{3}\right) \\
z_{2}(k+1) & =z_{2}(k)+\delta z_{1}(k)\left(1+u_{2}(k)\right)+O\left(\delta^{3}\right) .
\end{aligned}
$$

Accordingly, the control

$$
u_{1}^{\delta}(k)=-k_{1} \xi_{1}^{\delta}-k_{2} \xi_{2}^{\delta}
$$

with $k=2, k_{2}=-1.5$, stabilizes the transverse dynamics over $\Gamma^{\star}=\left\{x \in \mathbb{R}^{4}: \alpha(x)=\dot{\alpha}(x)=0\right\}=\left\{x \in \mathbb{R}^{4}: \xi_{1}^{\delta}=\xi_{2}^{\delta}=0\right\}$. In

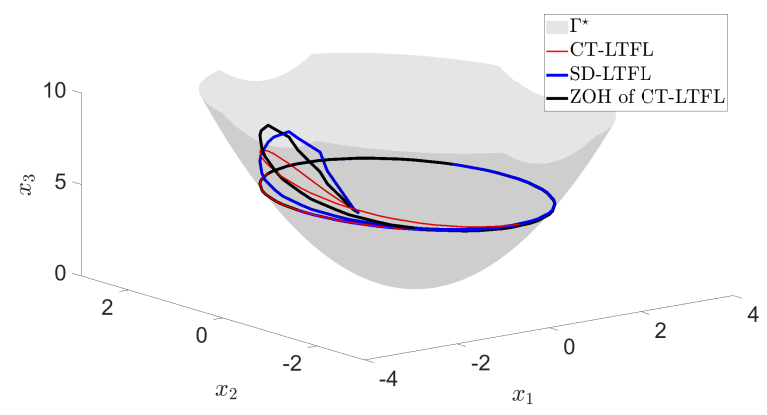

Fig. 3: States Evolution approaching $\Gamma^{\star}, \delta=0.4$

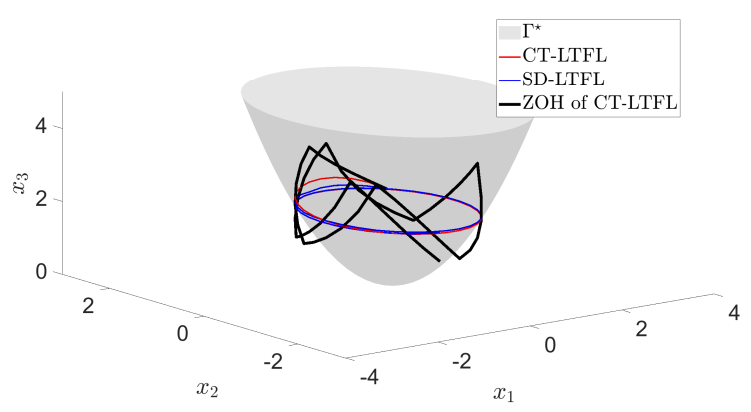

Fig. 4: Invariance of $\Gamma^{\star}, \delta=1$

Figure 3, the initial condition is $x_{0}=\operatorname{col}(1,2,2,0.5) \notin \Gamma^{\star}$ and the feedback (23) stabilizes the system to $\Gamma^{\star}$. Figure 4 illustrates that the invariance of $\Gamma^{\star}$ is preserved under the proposed feedback (23) while it fails when holding constant $(\mathrm{ZOH})$ the CT control; the initial state being $x=\operatorname{col}(1,1,2,0) \in \Gamma^{\star}$. In both simulations, the motion on $\Gamma^{\star}$ is free, i.e. $u_{2}(k)=0$.

\section{CONCLUSIONS}

A procedure to preserve transverse feedback linearization under digital control has been proposed. The first step of this iterative procedure leads to the redefinition of a linearizing output which comes out to depend in a polynomial way on the sampling period and allows for the design of a digital stabilizing control which outperforms simple zero-order holding of the continuous-time solution. Accordingly, attractivity and invariance of the set $\Gamma^{\star}$ is preserved under digital feedback with arbitrary orders of approximation. An exact solution to the (L)TFL-SD problem can be obtained using multi-rate sampling techniques, special care is needed when selecting the multirate orders on the input channels, this is deferred to a different work.

\section{ACKNOWLEDGEMENT}

The authors wish to thank the Associate Editor and the anonymous Reviewers for their helpful comments when preparing the final manuscript.

\section{REFERENCES}

[1] A. S. Shiriaev, "The notion of v-detectability and stabilization of invariant sets of nonlinear systems," Systems \& Control Letters, vol. 39, no. 5 , pp. $327-338,2000$.

[2] C. Nielsen and M. Maggiore, "On local transverse feedback linearization," SIAM Journal on Control and Optimization, vol. 47, no. 5, pp. 2227-2250, 2008.

[3] A. Hladio, C. Nielsen, and D. Wang, "Path following for a class of mechanical systems," IEEE Transactions on Control Systems Technology, vol. 21, no. 6, pp. 2380-2390, 2012.

[4] A. Doosthoseini and C. Nielsen, "Local nested transverse feedback linearization," Mathematics of Control, Signals, and Systems, vol. 27, no. 4, pp. 493-522, 2015.

[5] A. Astolfi and R. Ortega, "Immersion and invariance: A new tool for stabilization and adaptive control of nonlinear systems," IEEE Transactions on Automatic control, vol. 48, no. 4, pp. 590-606, 2003.

[6] M. Mattioni, S. Monaco, and D. Normand-Cyrot, "Immersion and invariance stabilization of strict-feedback dynamics under sampling," Automatica, vol. 76, pp. 78-86, 2017.

[7] A. Isidori, Nonlinear control systems. Springer Science \& Business Media, 2013.

[8] A. J. Krener, "On the equivalence of control systems and the linearization of nonlinear systems," SIAM Journal on Control, vol. 11, no. 4, pp. 670-676, 1973

[9] A. Banaszuk and J. Hauser, "Feedback linearization of transverse dynamics for periodic orbits," Systems \& control letters, vol. 26, no. 2, pp. 95-105, 1995.

[10] A. Arapostathis, B. Jakubczyk, H.-G. Lee, S. Marcus, and E. Sontag, "The effect of sampling on linear equivalence and feedback linearization," Systems and control letters, vol. 13, no. 5, pp. 373-381, 1989.

[11] S. Monaco and D. Normand-Cyrot, "Zero dynamics of sampled nonlinear systems," Systems \& control letters, vol. 11, no. 3, pp. 229-234, 1988.

[12] J. Grizzle and P. Kokotovic, "Feedback linearization of sampled-data systems," IEEE Transactions on Automatic Control, vol. 33, no. 9, pp. 857-859, 1988.

[13] S. Monaco and D. Normand-Cyrot, "Issues on nonlinear digital control," European Journal of Control, vol. 7, no. 2-3, pp. 160-177, 2001.

[14] J.-P. Barbot, S. Monaco, and D. Normand-Cyrot, "A sampled normal form for feedback linearization," Mathematics of Control, Signals and Systems, vol. 9, no. 2, pp. 162-188, 1996.

[15] C. Nielsen and M. Maggiore, "Output stabilization and maneuver regulation: A geometric approach," Systems \& control letters, vol. 55, no. 5, pp. 418-427, 2006.

[16] S. Monaco and D. Normand-Cyrot, "On nonlinear digital control," in Nonlinear systems. Springer, 1997, pp. 127-155.

[17] K. J. Åström, P. Hagander, and J. Sternby, "Zeros of sampled systems," Automatica, vol. 20, no. 1, pp. 31-38, 1984.

[18] J. I. Yuz and G. C. Goodwin, "On sampled-data models for nonlinear systems," IEEE transactions on automatic control, vol. 50, no. 10, pp. 1477-1489, 2005.

[19] C. Nielsen and M. Maggiore, "Maneuver regulation via transverse feedback linearization: Theory and examples," in Proceedings of the IFAC symposium on nonlinear control systems (NOLCOS), Stuttgart, Germany, 2004, pp. 59-66. 